\title{
Negative and positive affect are independently associated with patient-reported health status following percutaneous coronary intervention
}

\author{
Henneke Versteeg • Susanne S. Pedersen • \\ Ruud A. M. Erdman · Josephine W. I. van Nierop • \\ Peter de Jaegere $\cdot$ Ron T. van Domburg
}

Accepted: 2 July 2009/Published online: 19 July 2009

(C) The Author(s) 2009. This article is published with open access at Springerlink.com

\begin{abstract}
Purpose We examined the association between negative and positive affect and 12-month health status in patients treated with percutaneous coronary intervention (PCI) with drug-eluting stents.

Methods Consecutive PCI patients $(n=562)$ completed the Global Mood Scale at baseline to assess affect and the EuroQoL-5D (EQ-5D) at baseline and 12-month follow-up to assess health status.

Results Negative affect $[F(1,522)=17.14, P<.001]$ and positive affect $[F(1,522)=5.11, P=.02]$ at baseline were independent associates of overall health status at 12month follow-up, adjusting for demographic and clinical factors. Moreover, there was a significant interaction for negative by positive affect $[F(1,522)=6.11, P=.01]$. In domain-specific analyses, high negative affect was associated with problems in mobility, self-care, usual activities, pain/discomfort, and anxiety/depression with the risk being two to fivefold. Low positive affect was only associated with problems in self-care (OR: 8.14; 95\% CI: 1.85-35.9;
\end{abstract}

H. Versteeg $\cdot$ S. S. Pedersen $(\square)$

CoRPS-Center of Research on Psychology in Somatic diseases, Department of Medical Psychology, Tilburg University,

P.O. Box 90153, 5000 LE Tilburg, The Netherlands

e-mail: s.s.pedersen@uvt.nl

URL: http://www.tilburguniversity.nl/corps/

S. S. Pedersen - R. A. M. Erdman · J. W. I. van Nierop ·

P. de Jaegere - R. T. van Domburg

Department of Cardiology, Thoraxcenter, Erasmus Medical

Center, Rotterdam, The Netherlands

R. A. M. Erdman

Department of Medical Psychology and Psychotherapy, Erasmus Medical Center, Rotterdam, The Netherlands
$P=.006)$ and usual activities (OR: $1.87 ; 95 \%$ CI: $1.17-$ 3.00; $P=.009$ )

Conclusions Baseline negative and positive affect contribute independently to patient-reported health status 12 months post PCI. Positive affect moderated the detrimental effects of negative affect on overall health status. Enhancing positive affect might be an important target to improve patient-centered outcomes in coronary artery disease.

Keywords Coronary artery disease - Negative affect . Positive affect - Percutaneous coronary intervention . Self-reported health status

$\begin{array}{ll}\text { Abbreviations } \\ \text { CABG } & \text { Coronary artery bypass graft surgery } \\ \text { CAD } & \text { Coronary artery disease } \\ \text { EQ-5D } & \text { EuroQol-5 dimensions } \\ \text { EQ VAS } & \text { EuroQol visual analogue scale } \\ \text { GMS } & \text { Global Mood Scale } \\ \text { MI } & \text { Myocardial infarction } \\ \text { MVD } & \text { Multi-vessel disease } \\ \text { PCI } & \text { Percutaneous coronary intervention }\end{array}$

\section{Introduction}

Self-reported health status has gained recognition as an important outcome measure in patients with coronary artery disease (CAD) [1, 2], in part due to improved treatment options [3], but also due to subgroups of patients preferring health status and a reasonable quality of life over prolonged survival [4]. In addition, studies have 
shown that health status is associated with mortality and rehospitalization both in patients with CAD and chronic heart failure [5-7]. Hence, one of the goals of treatment is to enhance the patient's health status and well being in addition to prolonging survival [8].

However, there is a significant gap in our understanding of the determinants of health status outcomes in patients with $\mathrm{CAD}$, which knowledge is paramount in order to identify patients who may benefit from additional treatment and support [2]. Furthermore, the primary focus has been on the role of negative affect, such as depression and anxiety $[9,10]$, neglecting the role of positive affect, thereby failing to acknowledge that patients are not likely to characterize themselves by negative emotions alone [11].

Positive affect refers to feelings of joy, activity, and happiness [12] and is not merely the opposite of negative affect [13], since people can experience both types of affect at the same time [14]. Studies on the healthy elderly revealed that positive affect may influence perceived health status independent of and even more strongly than negative affect $[15,16]$.

The few studies that have examined the influence of positive and negative affect on health status simultaneously in CAD patients showed that positive affect moderated the detrimental effects of depressed affect on outcome [17-19]. These preliminary results suggest that negative and positive affect may both contribute uniquely to health status outcomes in CAD patients, but findings are limited and inconclusive about their independent value.

Hence, the objective of the current study was to determine the relative association of negative and positive affect assessed at baseline with 12-month overall health status and specific health status domains in patients treated with percutaneous coronary intervention (PCI) with drug-eluting stenting.

\section{Method}

Study design and participants

Consecutive patients treated with PCI with the paclitaxeleluting stent in the period from 15 February 2005 to 14 February 2006 in the Erasmus Medical Center Rotterdam, The Netherlands, comprised the sample for the current study. All surviving patients 30 days post PCI (referred to as baseline in the remainder of the paper) were approached by mail and asked to complete a set of psychological questionnaires. The same set of questionnaires was administered 12 months post PCI. If the questionnaire was not returned within 3 weeks, a reminder was sent to the patient together with a new questionnaire. All the patients were recommended to take clopidogrel for 6 months. The study protocol was approved by the medical ethics committee of the Erasmus Medical Center. All patients provided written informed consent, and the study was carried out in accordance with the Helsinki Declaration.

\section{Materials}

\section{Demographic and clinical variables}

Demographic variables included gender and age. Information on clinical variables (i.e., indication for PCI, multivessel disease, previous myocardial infarction (MI), previous PCI, previous coronary artery bypass graft surgery (CABG), hypertension, hypercholesterolemia, diabetes), and cardiac medication (i.e., aspirin, calcium antagonists, beta-blockers, ACE inhibitors, statins, diuretics) were obtained from the patients' medical records, except for smoking, which was assessed by means of self-report.

\section{Negative and positive affect}

Negative and positive affect were assessed with the Global Mood Scale [20]. The GMS comprises 10 negative affect terms (e.g., "helpless" and "insecure"), and 10 positive affect terms (e.g., "active" and "cheerful") that are rated on a 5-point Likert scale from 0 (not at all) to 4 (extremely). Hence, scores on both the negative affect and positive affect subscales range from 0 to 40 . A median split on the GMS scales was used to identify patients who reported high (above median) versus low (median or below) affect at baseline [21, 22]. For the negative affect subscale, the median score was 10 ; for the positive affect subscale, the median score was 22 . The GMS was initially validated in Belgian men with $\mathrm{CAD}$ and was found to be a psychometrically sound measure in terms of construct validity, internal consistency (Cronbach's $\alpha>.90$ ), and test-retest reliability $(r>.57)$ [20]. A number of studies by independent authors have confirmed the psychometric properties in cardiac patients $[19,23,24]$ and recently also in noncardiac adults from the working population [25]. In the current study, the internal consistency, as measured by Cronbach's $\alpha$, was high for both the negative $(\alpha=.91)$ and positive affect subscales $(\alpha=.93)$. Moreover, the GMS has been shown to be responsive to treatment-related changes in negative and positive affect among cardiac patients [21,23].

\section{Health status}

The EuroQol-5D (EQ-5D), a well-known generic measure of perceived health status developed by the EuroQol 
Group, was used as a measure of health status [26]. The measure consists of two parts: the EQ visual analogue scale (EQ VAS) and the EQ-5D descriptive system. On the EQ VAS, the respondent is asked to mark his/her current health state on a thermometer ranging from zero (worst imaginable health state) to 100 (best imaginable health state). This information is used as a quantitative measure of the respondent's overall perceived health status. The EQ-5D descriptive system comprises five questions tapping into the respondent's current health status in each of the following domains: Mobility, self-care, usual activities, pain/ discomfort, and anxiety/depression. Each question can be scored as: having no problems (level 1), some problems (level 2), or severe problems (level 3). In the present study, the EQ-5D levels were dichotomized into 'no problems' (i.e., level 1) versus 'problems' (i.e., levels 2 and 3) [27]. Dichotomization of patient-centered outcomes has been advocated previously in order to enhance clinical interpretability [28]. Based on the EQ-5D domains, it is also possible to calculate a utility score, a single summary index useful in cost utility analysis. However, in the current study, we did not use this score as we did not compare two treatment options and their associated costs. The EQ-5D is a brief and practical instrument with satisfactory validity and reliability for various diseases, including $\mathrm{CAD}$, and for the general population $[29,30]$.

\section{Statistical analyses}

Discrete variables were compared with the Chi-square test and continuous variables with Student's $t$-test for independent samples. To examine the impact of baseline affect on overall health status (EQ VAS) at 12-month follow-up, analysis of variance (ANOVA) was used with dichotomized negative and positive affect scores as between-subject factors. Analysis of covariance (ANCOVA) was performed to adjust for the potential confounding effects of baseline health status, age, gender, multi-vessel disease, cardiac history (defined as previous MI, PCI, or CABG), comorbidity (defined as hypertension, hypercholesterolemia, or diabetes), and smoking. To examine the impact of baseline affect on the five health status domains (EQ-5D descriptive system) at 12-month follow-up, univariable and multivariable logistic regression analyses were used. In multivariable analysis, we adjusted for the previously mentioned covariates. All variables were entered simultaneously in the multivariable models. In secondary analyses, the interaction effect, negative affect by positive affect was also included, to examine whether positive affect moderated the effect of negative affect on perceived health status.

All tests were two-tailed, and $P<.05$ was used to indicate statistical significance; in logistic regression analysis, odds ratios (OR) and their corresponding 95\% confidence intervals (CI) are reported. All data were analyzed using SPSS.17.0 for Windows (SPSS Inc., Chicago, Illinois).

\section{Results}

Participants versus nonparticipants on baseline characteristics

Of 1,238 eligible patients treated with PCI in the study period, 66 died within 30 days. The remaining 1,172 patients were asked to participate in the study, of which $870(74.2 \%)$ agreed. Participants $(n=870)$ were more likely to be older (mean $62.7 \pm 11.5$ vs. $60.2 \pm 13.4$; $P=.006)$ to have hypercholesterolemia ( $81.0 \%$ vs. $71.9 \%$; $P=.001)$, and to be prescribed aspirin $(92.6 \%$ vs. $87.1 \%$; $P=.005)$ compared to nonparticipants $(n=302)$. No other statistically significant differences were found between participants and nonparticipants on baseline characteristics. Of the 870 patients who agreed to participate, 67 did not fill in the GMS at follow-up. Of the remaining 803 patients, only 562 patients completed the entire EQ-5D descriptive system at follow-up and 533 the EQ VAS. Unfortunately, imputation of missing data was not an option because the EQ-5D descriptive system comprises only one question per domain, as does the EQ VAS. Hence, the main analyses focusing on the association between negative and positive affect and the EQ VAS are based on 533 patients, and the analysis related to the EQ5D descriptive system on 562 patients.

Baseline characteristics of the total sample and stratified by affect

Baseline characteristics for the total sample and stratified by negative and positive affect using a median split are presented in Table 1. Patients who scored high (above the median) on negative affect were more likely to be female (36\% vs. $20 \% ; P<.001)$, to have had a previous PCI (31\% vs. $20.3 \% ; P=.004)$, hypercholesterolemia $(86.8 \%$ vs. $78.8 \% ; P=.014)$, diabetes $(17.4 \%$ vs. $10.0 \% ; P=.011)$, to be smoking (28.9\% vs. $20.0 \% ; P=.014)$, and to be prescribed diuretics $(11.2 \%$ vs. $5.6 \% ; P=.017)$ compared to patients who scored low on negative affect. Patients who scored high on positive affect were less likely to have diabetes $(10.2 \%$ vs. $16.6 \% ; P=.03)$ compared to patients who scored low on positive affect. No other statistically significant differences were found on baseline demographics, clinical characteristics, and medication between groups. 
Table 1 Baseline characteristics for the total sample $(n=562)$ and stratified by affect

\begin{tabular}{|c|c|c|c|c|c|c|c|}
\hline & \multirow{2}{*}{$\begin{array}{l}\text { Total } \\
(n=562)\end{array}$} & \multicolumn{3}{|c|}{ GMS negative affect (cut-off $\geq 10$ ) } & \multicolumn{3}{|c|}{ GMS positive affect (cut-off $\geq 22$ ) } \\
\hline & & $\begin{array}{l}\text { Low } \\
(n=320)\end{array}$ & $\begin{array}{l}\text { High } \\
(n=242)\end{array}$ & $P$ & $\begin{array}{l}\text { Low } \\
(n=259)\end{array}$ & $\begin{array}{l}\text { High } \\
(n=303)\end{array}$ & $P$ \\
\hline \multicolumn{8}{|l|}{ Demographics } \\
\hline Female gender & $151(26.9)$ & $64(20.0)$ & $87(36.0)$ & $<.001 * * *$ & $77(29.7)$ & $74(24.4)$ & .16 \\
\hline Age, mean (SD) & $62.4(10.8)$ & $62.1(10.0)$ & $62.9(11.8)$ & .40 & $63.2(11.0)$ & $61.7(10.5)$ & .09 \\
\hline \multicolumn{8}{|l|}{ Clinical variables } \\
\hline MI as indication for PCI & $191(34.0)$ & $110(34.4)$ & $81(33.5)$ & .82 & $83(32.0)$ & $108(35.6)$ & .37 \\
\hline Multi-vessel disease & $261(46.4)$ & $155(48.4)$ & $106(43.8)$ & .27 & $117(45.2)$ & $144(47.5)$ & .58 \\
\hline Previous MI & $143(25.4)$ & $75(23.4)$ & $68(28.1)$ & .21 & $63(24.3)$ & $80(26.4)$ & .57 \\
\hline Previous PCI & $140(24.9)$ & $65(20.3)$ & $75(31.0)$ & $.004 * *$ & $69(26.6)$ & $71(23.4)$ & .38 \\
\hline Previous CABG & $44(7.8)$ & $24(7.5)$ & $20(8.3)$ & .74 & $22(8.5)$ & $22(7.3)$ & .59 \\
\hline Hypertension & $236(42.0)$ & $132(41.2)$ & $104(43.0)$ & .68 & $114(44.0)$ & $122(40.3)$ & .37 \\
\hline Hypercholesterolemia & $462(82.2)$ & $252(78.8)$ & $210(86.8)$ & $.014 *$ & $220(84.9)$ & $242(79.9)$ & .12 \\
\hline Diabetes & $74(13.2)$ & $32(10.0)$ & $42(17.4)$ & $.011^{*}$ & $43(16.6)$ & $31(10.2)$ & $.03 *$ \\
\hline Smoking & $134(23.8)$ & $64(20.0)$ & $70(28.9)$ & $.014 *$ & $70(27.0)$ & $64(21.1)$ & .10 \\
\hline \multicolumn{8}{|l|}{ Medication } \\
\hline Aspirin & $524(93.2)$ & $301(94.1)$ & $223(92.1)$ & .37 & $239(92.3)$ & $285(94.1)$ & .40 \\
\hline Calcium antagonists & $17(3.0)$ & $7(2.2)$ & $10(4.1)$ & .18 & $9(3.5)$ & $8(2.6)$ & .57 \\
\hline Beta-blockers & $359(63.9)$ & $206(64.4)$ & $153(63.2)$ & .78 & $165(63.7)$ & $194(64.0)$ & .94 \\
\hline ACE inhibitors & $211(37.5)$ & $112(35.0)$ & $99(40.9)$ & .15 & $95(36.7)$ & $116(38.3)$ & .70 \\
\hline Statins & $420(74.7$ & $245(76.6)$ & $175(72.3)$ & .25 & $192(74.1)$ & $228(75.2)$ & .76 \\
\hline Diuretics & $45(8.0)$ & $18(5.6)$ & $27(11.2)$ & $.017 *$ & $22(8.5)$ & 23 (7.6) & .69 \\
\hline
\end{tabular}

Results are presented as $n(\%)$, unless otherwise stated

$M I$ myocardial infarction, $P C I$ percutaneous coronary intervention, $C A B G$ coronary artery bypass graft surgery

$* P<.05 ; * * P<.01 ; * * * P<.001$

Negative and positive affect as determinants

of self-reported health status

\section{Overall health status (EQ VAS)}

For the 533 patients that completed the EQ VAS, the mean score at 12-month follow-up was $73.76( \pm 17.37)$. Univariable ANOVA showed a main effect for negative affect $[F(1,529)=104.58, P<.001]$ and for positive affect $[F(1,529)=19.93, P<.001]$ and a significant interaction for negative affect by positive affect $[F(1,529)=7.87$, $P=.005]$. Adjusting for covariates, the main and interaction effects of affect remained significant in ANCOVA. Patients with high negative affect reported significantly poorer overall health status than patients with low negative affect $[F(1,522)=17.14, P<.001]$. Patients with high positive affect reported significantly better overall health status than patients with low positive affect $[F(1$, $522)=5.11, P=.02]$. The significant interaction for negative affect by positive affect $[F(1,522)=6.11$, $P=.01]$ indicated that positive affect moderated the effect of negative affect on overall health status (Fig. 1). Of the

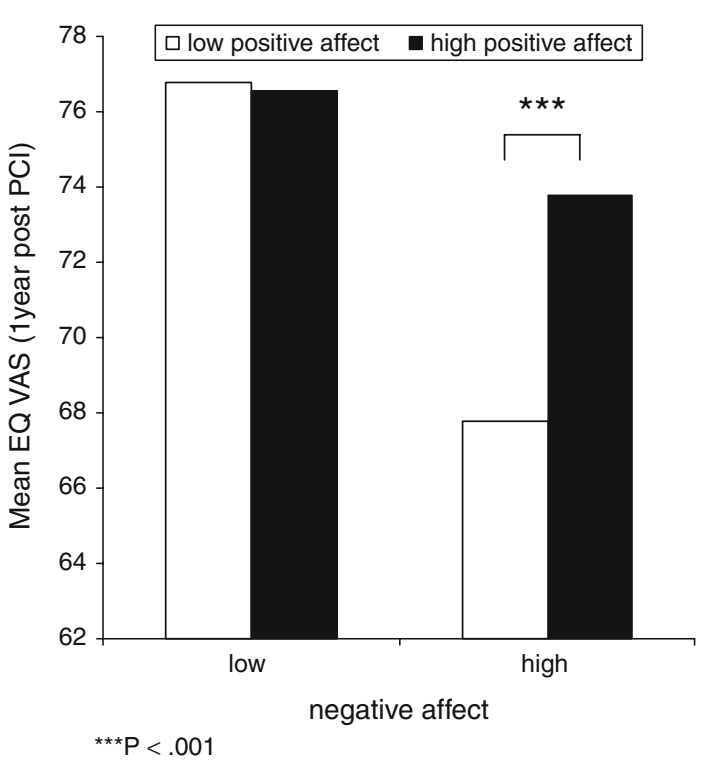

Fig. 1 Interaction of baseline negative affect with positive affect. *** $P<.001$. Means are adjusted for covariates (baseline EQ VAS, age, gender, MVD, cardiac history, comorbidity, and smoking) 
covariates, there was a main effect for baseline EQ VAS score $[F(1,522)=163.22, P<.001]$ and age $[F(1$, 522) $=5.28, P=.02]$.

\section{Domain-specific health status (EQ-5D descriptive system)}

Of the 562 patients that completed the EQ-5D descriptive system, $178(21.7 \%)$ patients reported mobility problems; $26(4.6 \%)$ patients problems with self-care; 160 (28.5\%) patients problems in usual activities; 215 (38.3\%) patients pain/discomfort; and 130 (23.1\%) patients symptoms of anxiety/depression, 12 months post PCI.

In univariable logistic regression analysis, entering the main effects for baseline negative affect and positive affect simultaneously, high negative affect had the strongest significant association with problems in all five domains: Mobility (OR: 4.17; 95\% CI: 2.79-6.24; $P<.001$ ); selfcare (OR: $10.85 ; 95 \%$ CI: 2.47-47.59; $P=.002$ ); usual activities (OR: 6.46; 95\% CI: 4.15-10.05; $P<.001$ ); pain/ discomfort (OR: 4.42; 95\% CI: 3.00-6.51; $P<.001$ ); and anxiety/depression (OR: 4.34; 95\% CI: 2.76-6.84; $P<.001$ ). Low positive affect had a weaker, but still statistically significant association with problems with self-care (OR: 5.12; 95\% CI: $1.48-17.77 ; P=.01$ ) and usual activities (OR: 1.94; 95\% CI: $1.26-2.98 ; P=.002$ ); the association with anxiety/depression problems was borderline significant (OR: 1.54; 95\% CI: .99-2.39; $P=.06$ ), showing that a high score on positive affect was protective of these problems. There was no significant association between positive affect and the other two dimensions, i.e., mobility and pain/discomfort.

Results of the multivariable analyses adjusted for baseline EQ-5D score, gender, age, multi-vessel disease, cardiac history, comorbidity, and smoking are shown in Table 2. High negative affect remained an independent associate of problems in all five domains, with ORs being two to fivefold (i.e., OR: 2.43 for pain/discomfort and OR: 5.33 for self-care). Low positive affect remained an independent associate of problems in self-care (OR: 8.14; $95 \%$ CI: $1.85-35.9 ; P=.006$ ) and in usual activities (OR: 1.87; 95\% CI: $1.17-3.00 ; P=.009)$ in adjusted analysis. There was no significant interaction for negative affect by positive affect for each of the five domains.

\section{Discussion}

Self-reported health status is an important outcome measure in patients with CAD, but little is known about its determinants [2]. Until now, data are limited and inconclusive about the association of affect, particularly positive affect, with health status in CAD patients. Results of the current study demonstrated that baseline negative and

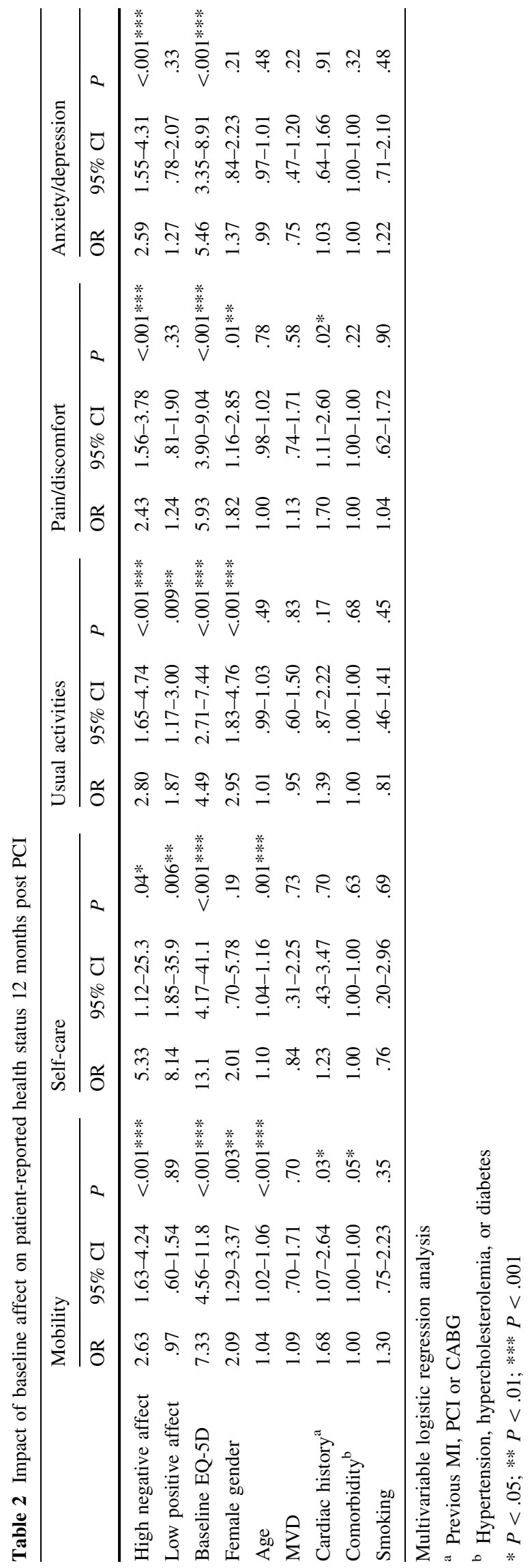


positive affect are independently associated with health status 12 months post PCI, adjusting for demographic and clinical factors and baseline health status.

After baseline health status, negative affect was the most important independent associate of 12-month overall health status in this study, followed by positive affect and age. These results are in line with the findings of Dua [31], but inconsistent with more recent findings in the healthy elderly showing stronger associations between health status and positive affect $[15,16]$. An explanation for this inconsistency could be the diversity of health status indices used across studies [16], as research indicates that positive and negative affect generally are related to different types of health status. Watson [32] reported that negative affect is strongly associated with perceived stress and health complaints, while positive affect is highly related to social activity and exercise. To investigate this, we additionally analyzed the influence of both types of affect on specific health status domains; that is physical functioning (mobility and self-care), social functioning (usual activities), mental functioning (anxiety/depression), and pain/ discomfort [29]. The results showed that the incidence of problems in each of the health status domains at 12-month follow-up were significantly higher in patients reporting high negative affect versus those reporting low negative affect at baseline, with the associated risk being two to fivefold adjusting for demographic and clinical factors, and positive affect. A low baseline score on positive affect was associated with problems in self-care and usual activities only, with ORs of 8.14 and 1.87 , respectively. These results support the notion that positive and negative affects are not merely the opposite ends of a continuum [14], and point to the usefulness of including separate positive and negative affect scales in health outcomes research.

In the current study, we were also able to replicate that positive affect moderated the detrimental effect of negative affect on overall health status in CAD patients [17, 18]. Patients reporting high negative and positive affect reported a much better overall health status compared to patients reporting high negative affect and low positive affect. This indicates that positive affect might play a protective role for health status in the presence of negative affect. In line with this finding, Fredrickson and Levenson [33] demonstrated that positive emotions can dampen the physiological reactivity evoked by negative emotions and interrupt or shorten the damaging impact that this reactivity has on the cardiovascular system.

Research on the specific mechanisms that link affect and health outcomes is not yet clear [12]. Affect may influence health directly by eliciting changes in neuroendocrine, inflammatory, and immune processes [34-36]. Alternatively, affect might influence people's perceptions of and decisions regarding their health [37]. Patients reporting high negative affect may be more likely to notice and attend to bodily sensations and to interpret them as painful or pathological, while patients reporting high positive affect may interpret ambiguous sensations in a more positive way [35, 38]. In addition, affect can influence health-promoting behaviors and adherence to treatment $[39,40]$. These potential pathways remain speculative as they are yet to be tested empirically in future research.

More research is warranted to investigate the predictive role of positive affect on hard medical endpoints in CAD, including mortality. In contrast to the bulk of research linking negative emotional states to clinical outcomes, a paucity of studies have evaluated the impact of positive psychological factors [41]. Up to now, only one study has demonstrated that reduced positive affect was a significant independent predictor of adverse clinical events following PCI [11]. These and the present results indicate that CAD patients might benefit from behavioral interventions, like somatic relaxation training and mindfulness-based stress reduction, focusing on enhancing positive affect, besides reducing negative affect $[42,43]$.

The results of the current study should be interpreted with some caution due to the following limitations. First, since the study was observational, no firm conclusions can be drawn regarding a cause-and-effect relationship between affect and health status in CAD patients. However, it would be interesting to examine this in a clinical trial manipulating affect. Second, we had no information on left ventricular ejection fraction as this was not consistently collected, but we did adjust for multi-vessel disease and previous cardiac history, as measures of disease severity. Finally, we used a generic measure of health status, which may be less clinically sensitive than a disease-specific measure [44].

Strengths of the study include its prospective design with a follow-up period of 12 months and the relatively large sample size. In addition, the majority of research on the role of psychological factors in CAD has been conducted in MI patients rather than in PCI patients and focused on the studying of negative affect, such as depression. Given the paucity and inconclusiveness of research on affect and health status in CAD, the current findings add to our understanding of the influence of emotions on patient-centered health outcomes in cardiac patients, indicating that negative affect and positive affect should be studied in concert.

In conclusion, the present study showed that baseline negative affect is a significant independent associate of impaired 12-month health status in CAD patients, adjusting for previously identified risk factors, including gender, age, cardiac history, and comorbid conditions. Low positive affect was associated with impaired health status above and beyond negative affect and demographic and clinical risk 
factors, particularly for problems in self-care and usual activities. These results indicate that positive affect might play a protective role for health status and emphasize the importance of studying positive affect in CAD in its own right. Given these findings, cardiac rehabilitation should not only target the reduction of negative emotions and distress but also seek to enhance positive affect in individual patients.

Open Access This article is distributed under the terms of the Creative Commons Attribution Noncommercial License which permits any noncommercial use, distribution, and reproduction in any medium, provided the original author(s) and source are credited.

\section{References}

1. Bullinger, M. (2002). Assessing health related quality of life in medicine. An overview over concepts, methods and applications in international research. Restorative Neurology and Neuroscience, 20(3-4), 93-101.

2. Krumholz, H. M., Peterson, E. D., Ayanian, J. Z., Chin, M. H., DeBusk, R. F., Goldman, L., et al. (2005). Report of the National Heart, Lung, and Blood Institute working group on outcomes research in cardiovascular disease. Circulation, 111(23), 31583166.

3. Hoffman, S. N., TenBrook, J. A., Wolf, M. P., Pauker, S. G., Salem, D. N., \& Wong, J. B. (2003). A meta-analysis of randomized controlled trials comparing coronary artery bypass graft with percutaneous transluminal coronary angioplasty: One- to eight-year outcomes. Journal of the American College of Cardiology, 41(8), 1293-1304.

4. Stanek, E. J., Oates, M. B., McGhan, W. F., Denofrio, D., \& Loh, E. (2000). Preferences for treatment outcomes in patients with heart failure: Symptoms versus survival. Journal of Cardiac Failure, 6(3), 225-232.

5. Lenzen, M. J., Scholte op Reimer, W. J. M., Pedersen, S. S., Boersma, E., Maier, W., Widimsky, P., et al. (2007). The additional value of patient-reported health status in predicting 1-year mortality after invasive coronary procedures: A report from the Euro Heart Survey on coronary revascularisation. Heart, 93(3), 339-344.

6. Soto, G. E., Jones, P., Weintraub, W. S., Krumholz, H. M., \& Spertus, J. A. (2004). Prognostic value of health status in patients with heart failure after acute myocardial infarction. Circulation, 110(5), 546-551.

7. Rumsfeld, J. S., MaWhinney, S., McCarthy, M., Shroyer, A. L., VillaNueva, C. B., O’Brien, M., et al. (1999). Health-related quality of life as a predictor of mortality following coronary artery bypass graft surgery. Participants of the department of veterans affairs cooperative study group on processes, structures, and outcomes of care in cardiac surgery. Journal of the American Medical Association, 281(14), 1298-1303.

8. Benzer, W., Höfer, S., \& Oldridge, N. B. (2003). Health-related quality of life in patients with coronary artery disease after different treatments for angina in routine clinical practice. Herz, 28(5), 421-428.

9. Ruo, B., Rumsfeld, J. S., Hlatky, M. A., Liu, H., Browner, W. S., \& Whooley, M. A. (2003). Depressive symptoms and healthrelated quality of life: The heart and soul study. Journal of the American Medical Association, 290(2), 215-221.

10. Höfer, S., Benzer, W., Alber, H., Ruttmann, E., Kopp, M., Schüssler, G., et al. (2005). Determinants of health-related quality of life in coronary artery disease patients: A prospective study generating a structural equation model. Psychosomatics, 46(3), 212-223.

11. Denollet, J., Pedersen, S. S., Daemen, J., de Jaegere, P., Serruys, P. W., \& van Domburg, R. T. (2008). Reduced positive affect (anhedonia) predicts major clinical events following implantation of coronary-artery stents. Journal of Internal Medicine, 263(2), 203-211.

12. Pressman, S. D., \& Cohen, S. (2005). Does positive affect influence health? Psychological Bulletin, 131(6), 925-971.

13. Tellegen, A., Watson, D., \& Clark, L. A. (1999). On the dimensional and hierarchical structure of affect. Psychological Science, 10(4), 297-303.

14. Larsen, J. T., McGraw, A. P., \& Cacioppo, J. T. (2001). Can people feel happy and sad at the same time? Journal of Personality and Social Psychology, 81(4), 684-696.

15. Benyamini, Y., Idler, E. L., Leventhal, H., \& Leventhal, E. A. (2000). Positive affect and function as influences on self-assessments of health: Expanding our view beyond illness and disability. The Journals of Gerontology Series B: Psychological Sciences and Social Sciences, 55, 107-116.

16. Winter, L., Lawton, M. P., Langston, C. A., Ruckdeschel, K., \& Sando, R. (2007). Symptoms, affects, and self-rated health: Evidence for a subjective trajectory of health. Journal of Aging and Health, 19(3), 453-469.

17. Sullivan, M. D., LaCroix, A. Z., Russo, J. E., \& Walker, E. A. (2001). Depression and self-reported physical health in patients with coronary disease: Mediating and moderating factors. Psychosomatic Medicine, 63(2), 248-256.

18. Martens, E. J., Smith, O. R. F., \& Denollet, J. (2007). Psychological symptom clusters, psychiatric comorbidity and poor selfreported health status following myocardial infarction. Annals of Behavioral Medicine, 34(1), 87-94.

19. Spindler, H., Denollet, J., Kruse, C., \& Pedersen, S. S. (2009). Positive affect and negative affect correlate differently with distress and health-related quality of life in patients with cardiac conditions: Validation of the Danish global mood scale. Journal of Psychosomatic Research, 67, 57-65.

20. Denollet, J. (1993). Emotional distress and fatigue in coronary heart disease: The Global Mood Scale (gms). Psychological Medicine, 23(1), 111-121.

21. Denollet, J., \& Brutsaert, D. L. (1995). Enhancing emotional well-being by comprehensive rehabilitation in patients with coronary heart disease. European Heart Journal, 16(8), 1070-1078.

22. Pelle, A. J., Pedersen, S. S., Szabó, B. M., \& Denollet, J. (2009). Beyond Type D personality: Reduced positive affect (anhedonia) predicts impaired health status in chronic heart failure. Quality of Life Research (in press). doi: 10.1007/s11136-009-9485-z.

23. Hevey, D., McGee, H. M., \& Horgan, J. (2004). Responsiveness of health-related quality of life outcome measures in cardiac rehabilitation: Comparison of cardiac rehabilitation outcome measures. Journal of Consulting and Clinical Psychology, 72(6), $1175-1180$.

24. Lowe, R., Norman, P., \& Bennett, P. (2000). Coping, emotion and perceived health following myocardial infarction: Concurrent and predictive associations. British Journal of Health Psychology, 5(4), 337-350.

25. Denollet, J., \& De Vries, J. (2006). Positive and negative affect within the realm of depression, stress and fatigue: The two-factor distress model of the global mood scale (gms). Journal of Affective Disorders, 91(2-3), 171-180.

26. EuroQol-group. (1990). Euroqol-a new facility for the measurement of health-related quality of life. The EuroQol group. Health Policy, 16(3), 199-208.

27. Kind, P., Dolan, P., Gudex, C., \& Williams, A. (1998). Variations in population health status: Results from a United Kingdom 
National Questionnaire survey. British Medical Journal, 316(7133), 736-741.

28. Rumsfeld, J. S., Magid, D. J., Plomondon, M. E., Sales, A. E., Grunwald, G. K., Every, N. R., et al. (2003). History of depression, angina, and quality of life after acute coronary syndromes. American Heart Journal, 145(3), 493-499.

29. Brooks, R. (1996). EuroQol: The current state of play. Health Policy, 37(1), 53-72.

30. Schweikert, B., Hahmann, H., \& Leidl, R. (2006). Validation of the EuroQol questionnaire in cardiac rehabilitation. Heart, 92(1), 62-67.

31. Dua, J. K. (1994). Comparative predictive value of attributional style, negative affect, and positive affect in predicting selfreported physical health and psychological health. Journal of Psychosomatic Research, 38(7), 669-680.

32. Watson, D. (1988). Intraindividual and interindividual analyses of positive and negative affect: Their relation to health complaints, perceived stress, and daily activities. Journal of Personality and Social Psychology, 54(6), 1020-1030.

33. Fredrickson, B. L., \& Levenson, R. W. (1998). Positive emotions speed recovery from the cardiovascular sequelae of negative emotions. Cognition and Emotion, 12(2), 191-220.

34. Steptoe, A., O’Donnell, K., Badrick, E., Kumari, M., \& Marmot, M. (2008). Neuroendocrine and inflammatory factors associated with positive affect in healthy men and women: The Whitehall II Study. American Journal of Epidemiology, 167(1), 96-102.

35. Cohen, S., Doyle, W. J., Turner, R. B., Alper, C. M., \& Skoner, D. P. (2003). Emotional style and susceptibility to the common cold. Psychosomatic Medicine, 65(4), 652-657.

36. Stone, A. A., Marco, C. A., Cruise, C. E., Cox, D. S., \& Neale, J. M. (1996). Are stress-induced immunological changes mediated by mood? A closer look at how both desirable and undesirable daily events influence siga antibody. International Journal of Behavioral Medicine, 3(1), 1-13.

37. Salovey, P., Rothman, A. J., Detweiler, J. B., \& Steward, W. T. (2000). Emotional states and physical health. American Psychologist, 55(1), 110-121.

38. Watson, D., \& Pennebaker, J. W. (1989). Health complaints, stress, and distress: Exploring the central role of negative affectivity. Psychological Review, 96(2), 234-254.

39. Peterson, C. (1988). Explanatory style as a risk factor for illness. Cognitive Therapy and Research, 12(2), 119-132.

40. Salovey, P., \& Birnbaum, D. (1989). Influence of mood on health-relevant cognitions. Journal of Personality and Social Psychology, 57(3), 539-551.

41. Rozanski, A., Blumenthal, J. A., Davidson, K. W., Saab, P. G., \& Kubzansky, L. (2005). The epidemiology, pathophysiology, and management of psychosocial risk factors in cardiac practice: The emerging field of behavioral cardiology. Journal of the American College of Cardiology, 45(5), 637-651.

42. Jain, S., Shapiro, S. L., Swanick, S., Roesch, S. C., Mills, P. J., Bell, I., et al. (2007). A randomized controlled trial of mindfulness meditation versus relaxation training: Effects on distress, positive states of mind, rumination, and distraction. Annals of Behavioral Medicine, 33(1), 11-21.

43. Nyklicek, I., \& Kuijpers, K. F. (2008). Effects of mindfulnessbased stress reduction intervention on psychological well-being and quality of life: Is increased mindfulness indeed the mechanism? Annals of Behavioral Medicine, 35(3), 331-340.

44. Thompson, D. R., \& Yu, C. M. (2003). Quality of life in patients with coronary heart disease-I: Assessment tools. Health and Quality of Life Outcomes, 1, 42. 